\title{
Anmerkungen zum Eckpunktepapier der Arbeitsgruppe Sicherungsverwahrung Berlin-Brandenburg
}

\author{
Hans-Ludwig Kröber
}

Eingegangen: 4. März 2011 / Angenommen: 17. März 2011 / Online publiziert: 13. April 2011

(C) Springer-Verlag 2011

Die Herausgeber haben sich zur Veröffentlichung des vorstehenden Eckpunktepapiers der Arbeitsgruppe Sicherungsverwahrung Berlin-Brandenburg entschlossen, weil hier entgegen der allgemeinen Tendenz, immer mehr auf Sicherung und Ausgrenzung zu setzen, seitens der Justizverwaltungen zweier Bundesländer das alte, verfassungskonforme Ziel der Resozialisierung wieder in den Vordergrund gerückt wird. Verdeutlicht wird, dass es bei der Reform der Sicherungsverwahrung nicht nur um eine Formalanpassung an Straßburger Vorgaben zur Größe von Zelle und Fernsehschirm und zur Distanz gegenüber Strafgefangenen gehen kann, sondern um den Umgang mit den Verurteilten, um deren Behandlung im allgemeinen Sinne des Wortes.

Wichtig dabei ist, dass Behandlungsstandards für den Strafvollzug und den Vollzug der Sicherungsverwahrung vorgegeben werden, die keineswegs nur zukünftig irgendwann anzustreben, sondern in vielen Aspekten sofort umsetzbar sind [1]. Sicherlich gibt es Haftanstalten und Aufsichtsbehören, die den Umgang mit Langstrafern und Sicherungsverwahrten bereits jetzt in dieser oder einer ähnlichen Weise organisieren. Gleichwohl gibt es immer noch Regionen, in denen die Tatsache, dass ein Verurteilter zudem noch für die Sicherungsverwahrung vorgesehen ist, zur Lähmung vollzuglicher Aktivitäten führt, die der Resozialisierung des Insassen dienen könnten: therapeutische Maßnahmen, qualifizierende Maßnahmen, mögliche Lockerungen. Ein ganz zentraler Gedanke des Eckpunktepapiers ist, dass die Therapie möglichst früh angestrebt werden soll und dass ein erstes Ziel darin liegt, durch eine erfolgrei-

Univ.-Prof. Dr. med. H.-L. Kröber $(\bowtie)$

Institut für Forensische Psychiatrie, Charité -

Universitätsmedizin Berlin, Oranienburger Str. 285 (Haus 10),

13437 Berlin, Deutschland

E-Mail: Hans-Ludwig.Kroeber@charite.de che Gestaltung der Haftzeit den Vollzug der Maßregel der Sicherungsverwahrung überflüssig zu machen. Hier geht es also darum, den Verurteilten rechtzeitig für die Kooperation in der Sozialtherapie oder andere, funktionierende Therapieprogramme zu gewinnen und dann diese Therapie mit ihm durchzuführen. Es gibt hier keine naiven Erwartungen, dies könnte stets gelingen, vielleicht nicht einmal in der Mehrzahl der Fälle: Aber der therapeutische Fokus hat zeitlich vorn zu liegen und nicht hinten (und schon gar nicht erst nach der Entlassung).

In manchen Regionen wird zudem die Sozialtherapie von Gefangenen als eine Risikoetappe, gerüchteweise dann als Falle, beschrieben: Die Wahrscheinlichkeit sei hoch, dass man da scheitere und in den Normalvollzug zurückgeschickt werde. Es ist also nach wie vor auch erforderlich, dass die sozialtherapeutischen Einrichtungen sich prüfen, ob sie die Wandlung von der angebots- zur nachfrageorientierten Einrichtung geschafft haben und ob sie genügend adäquate Methoden für ihre besondere Klientel vorweisen - auch für deren Vielfalt hinsichtlich Persönlichkeit, sozialer Vorerfahrung und Intelligenz. Das Eckpunktepapier zeichnet sich dadurch aus, dass es nicht nur eine einzige therapeutische Option kennt, sondern für eine Anpassung der Angebote an den Bedarf wirbt.

Das Eckpunktepapier legt einen zweiten Schwerpunkt auf das Ende der Unterbringungszeit, und hier heißt das Stichwort „Übergangsmanagement“. Es umfasst in etwa einen Zeitraum von 2 bis 3 Jahren und dient der besonders sorgfältigen Reintegration (oftmals sogar der erstmaligen Integration) des zu Entlassenden in einen stützenden, hinreichend befriedigenden und eben auch sozial kontrollierenden sozialen Empfangsraum. Das Übergangsmanagement zur Reintegration (das natürlich immer „case management" ist, also - möglichst mit personeller Konstanz um den jeweils einzelnen Verurteilten herum aufgebaut 
werden muss), wird gegenwärtig bei den Untergebrachten, die aufgrund Straßburger Urteils sehr schnell entlassen werden müssen, forciert erlernt. Tatsächlich haben die meisten Haftanstalten in dieser Disziplin noch Lern- und Trainingsbedarf, allemal, wenn man es mit der Qualität der Entlassungsvorbereitung im psychiatrischen Maßregelvollzug vergleicht. Gegenwärtig sind viele Einrichtungen, in denen Sicherungsverwahrung vollzogen wird, gar nicht gewohnt und bereit, überhaupt Lockerungen zu gewähren. Es fehlen zudem Mitarbeiter für die Begleitung, und es fehlt oft auch das sozialarbeiterische Personal, das in dieser Tätigkeit der Resozialisierung erfahren und mit Nachsorgeeinrichtungen, Bewährungshilfe, Ämtern vernetzt ist. Es ist aber falsch, mögliche Entlassungen dadurch zu verhindern, dass überhaupt keine Wege in die Freiheit vorgesehen sind, die weniger steil sind als die abrupte Entlassung aus dem ungelockerten geschlossenen Vollzug. Dies gilt umso mehr, wenn man alle vorangehenden Anstrengungen, die Einstellungen und Verhaltensbereitschaften des Gefangenen nachhaltig zu verändern, wieder zunichte macht, indem man ihm nur die Perspektive der Hospitalisierung lässt. Also: Wenn die Einschätzung der akuten Gefährlichkeit es zulässt, müssen gerade beim Vollzug der Maßregel Lockerungen gewährt, fachkundig unterstützt und genutzt werden, um außerhalb der Vollzugseinrichtung ein soziales Lebensfeld zu erschlieBen und in Beziehungen, Aktivitäten und Kenntnissen zu konkretisieren.

Ein kleiner Mangel des Eckpunktepapiers, der hoffentlich nicht den Weg in bindende Vorschriften findet, liegt in der geforderten personellen Aufblähung der externen Begutachtung. Schon jetzt dienen diese Pflichtbegutachtungen, so die in Hessen vorgeschriebene Doppelbegutachtung vor Lockerungen, nicht selten der Verantwortungsverschiebung und der massiven Verlangsamung von Reintegrationsprozessen. Außerdem bremst es den notwendigen Prozess, innerhalb der Einrichtungen noch mehr an kriminaldiagnostischer Kompetenz aufzubauen. Durch die Ausweitung der Begutachtungsnachfrage wird der Kreis der vermeintlich Sachverständigen auf solche Personen ausgeweitet, die nicht hinreichend qualifiziert sind, im Regelfall für Verbleib plädieren und ansonsten Verwirrung stiften. Das Land Brandenburg forderte zunächst, es müsse stets ein Dreiergremium unter Einschluss eines Sozialpädagogen gutachten (also - wenn jeder die Aufgabe ernst nimmt und nicht Trittbrettfahrer spielt: dreifacher Zeitaufwand, dreifache Kosten, aber stets bestenfalls nur ein richtiges Ergebnis). In der Endfassung ist nun die Rede von einem ,interdisziplinär arbeitenden Gutachterteam“. Dies sollte man so verstehen, dass das Land ein Team von psychiatrischen, psychologischen, kriminologischen Gutachtern kennt, die in Qualitätszirkeln und steter Fortbildung die Beachtung wissenschaftlicher Standards absichern, die je einzeln betraut werden können und die in der Erläuterung ihres Gutachtens interdisziplinär mit den Fachdiensten und dem Vollzugsdienst zusammenarbeiten.

Insgesamt ist das Eckpunktepapier kein radikales Konzept zur Veränderung der Sicherungsverwahrung, und es kann die charakteristischen psychologischen Probleme, die mit dieser Maßregel verknüpft sind, natürlich auch nicht beseitigen. Weil es aber sehr pragmatisch von den bestehenden Möglichkeiten und Strukturen ausgeht, kann es Mindeststandards beschreiben. Würden diese überall erreicht und eingehalten, wäre dies bereits ein großer Fortschritt, eben nicht in Richtung Ausstoßung, sondern in die Richtung, möglichst viele doch noch für ein Leben in sozialer Verantwortung zu gewinnen.

Interessenkonflikt Der Autor gibt keinen Interessenkonflikt an.

\section{Literatur}

1. Preusker H, Maelicke B, Flügge C (Hrsg) (2010) Das Gefängnis als Risiko-Unternehmen. Nomos, Baden-Baden 